\title{
VISUAL FIELD DEFECTS AND THE RISK OF MOTOR VEHICLE COLLISIONS AMONG PATIENTS WITH GLAUCOMA
}

\author{
Gerald McGwin, Jr. ${ }^{1}$, Aiyuan Xie ${ }^{2}$, Andrew Mays ${ }^{2}$, Wade Joiner ${ }^{2}$, \\ Dawn K. DeCarlo ${ }^{3}$, Tyler Andrew Hall ${ }^{2}$, Cynthia Owsley ${ }^{2}$ \\ ${ }^{1}$ Departments of Ophthalmology and Epidemiology \\ ${ }^{2}$ Department of Ophthalmology \\ University of Alabama at Birmingham \\ Birmingham, Alabama, USA \\ E-mail: mcgwin@uab.edu \\ ${ }^{3}$ College of Optometry \\ Nova Southeastern University \\ Ft. Lauderdale, Florida, USA
}

\begin{abstract}
Summary: This study sought to evaluate the association between visual field defects in the central $24^{\circ}$ field and motor vehicle collisions (MVC) among patients with glaucoma. A case-control study was conducted on glaucoma patients aged 55 and older being treated at three university-affiliated eye care practices. Cases were patients who sustained a police-reported motor vehicle collision (MVC); controls were those who did not experience a MVC. For each patient, an Advanced Glaucoma Intervention Study (AGIS) score was calculated on automated visual fields collected with the 24-2 or 30-2 programs. Additionally, demographic, behavioral, driving and clinical characteristics were obtained via chart abstractions and a patient telephone survey. With respect to the better eye AGIS score (defined according to AGIS score), compared to patients with no visual field defect, those with severe defects (scores 12-20) had an increased risk of MVC (odds ratio [OR] 3.2, 95\% confidence interval [CI] 0.9-10.4), although this association was not statistically significant $(\mathrm{p}=0.0576)$. Moderate $(6-11)$ or minor field defects (1-5) in the better eye were not associated with the risk of crash involvement. For the worse eye, patients with moderate and severe field defects were at significantly increased risk of a MVC (OR 3.6, 95\% CI 1.4-9.4 and OR 4.4, 95\% CI 1.6-12.4 respectively) compared to those with no defects. Minor field defects in the worse eye did not increase MVC risk (OR 1.3, 95\% CI 0.5-3.4). Glaucoma patients with moderate or severe visual field impairment in the central $24^{\circ}$ radius field in the worse functioning eye are at increased risk for involvement in a vehicle crash.
\end{abstract}

\section{INTRODUCTION}

Glaucoma is a chronic progressive optic neuropathy characterized by changes of the optic disk, thinning of the retinal nerve fiber layer, and gradual loss of visual function beginning in the peripheral field. An estimated 6.7 million people worldwide are bilaterally blind from glaucoma and another 67 million are affected by glaucoma, with about half of those who are unaware they have the disease (Quigley, 1996). The loss of peripheral vision due to glaucoma is associated with decreased health-related quality of life (Gutierrez et al., 1997). 
Motor vehicle collisions (MVC) are among the most potentially adverse mobility-related outcomes previously reported to be associated with a diagnosis of glaucoma (Hu et al., 1998; Johnson \& Keltner, 1983; Owsley, McGwin \& Ball, 1998), although not all agree (McCloskey et al. 1994). Previous studies had design features that lead one to question the validity of the conclusions including the reliance on a diagnosis definition based on self-report of glaucoma (Hu et al., 1998; Johnson \& Keltner, 1983; Owsley, McGwin \& Ball, 1998), failure to take driving exposure into account when assessing crash risk (Hu et al., 1998), and only a small number of glaucoma cases in the study sample (Hu et al., 1998; Owsley, McGwin \& Ball, 1998). Recently we reported a cohort study that overcame these methodological problems finding that adults aged $\geq 55$ years with a diagnosis of glaucoma drive at least as safely as older persons without glaucoma (McGwin et al., 2004).

What was not addressed in this earlier report was whether the subpopulation of glaucomatous drivers with visual field impairment is at increased risk for vehicle crash involvement as compared to those with no field loss. This issue has clear clinical and policy relevance since information about how severe visual field loss has to be before negatively impacting driver safety is not well understood.

\section{METHODS}

\section{Study Cohort}

The study cohort consisted of individuals aged 55 and older who had been seen at least once between January 1994 and December 1995 in any of three university-affiliated ophthalmology and optometry practices specializing in the diagnosis and treatment of glaucoma. The International Classification of Diseases, Ninth Revision, Clinical Modification (ICD-9-CM) codes 365.1 and 365.2 were used to identify all potentially eligible glaucoma patients seen at each of these locations. The medical records of each potentially eligible patient were abstracted to verify the diagnosis of glaucoma. Patients were excluded if: (1) their primary cause of visual impairment was an ocular disorder other than glaucoma (e.g., macular degeneration, diabetic retinopathy, or clinically significant cataract where surgery was recommended; persons with diagnoses of refractive error, dry eye, and early cataract were eligible for the study), (2) automated visual field data (either a 30-2 or 24-2 test) for both eyes was not in the medical record during the study period; and (3) patients were not legally licensed to drive in the State of Alabama. Information on licensure status was obtained by cross-referencing each subject's demographic and residential information obtained from the medical record with the Alabama Department of Public Safety (ADPS) database.

\section{Data Collection}

In addition to confirming the diagnosis of glaucoma, medical records were also used to obtain information on glaucoma medication use, best-corrected visual acuity OD and OS, and visual fields OD and OS. All patient visits during the time period January 1994 through December 1995 were abstracted. The visual field reports were then used to calculate a visual field defect score for each eye based on the Advanced Glaucoma Intervention Study (AGIS) scoring system (Advanced Glaucoma Intervention Study, 1994). AGIS scores were categorized into four categories based upon previously described cut points (Advanced Glaucoma Intervention Study, 
1994): no defect (score 0), mild defect (scores 1-5), moderate defect (scores 6-11), and severe defect (scores 12-20).

To obtain additional information on demographic, driving, general health, smoking and alcohol use, a telephone survey was conducted between February and June 2000. Demographic information was gathered using standard questions regarding age, gender, and race. History of cigarette smoking and alcohol use was assessed with standard questions (Owsley, et al., 2002). The Short Portable Mental Status Questionnaire (Pfeiffer, 1975), modified for telephone administration (Roccaforte et al., 1994), was used to assess cognitive status. Respondents were asked to respond to a general health questionnaire and questions on driving habits using the year 1995 as the reference point. The Driving Habits Questionnaire (DHQ) (Owsley et al., 1999), previously shown to be reliable and valid among older drivers, was used to collect information on driving exposure defined in terms of estimated weekly mileage (Murakami \& Wagner, 1997). The DHQ was also used to calculate a driving avoidance score to estimate the extent to which a respondent avoided certain driving situations that are known to be especially problematic for older drivers. Items addressed driving at night, in fog, in the rain, while alone, during rush hour, on the highway/freeway, with children, in high density traffic, when passing other cars, when changing lanes, when making left hand turns at intersections, and parallel parking. The possible responses were "Always," "Often," "Sometimes," "Rarely," or "Never." For analytic purposes, subjects who reported "Always" or "Often" avoiding a specific situation were defined as avoiders and those who reported "Sometimes," "Rarely," or "Never" were defined as nonavoiders. A composite variable to reflect overall driving avoidance was created by summing the binary avoider/non-avoider variables. Given the 12 situations, the composite variable had a range of 12 (minimum: 0; maximum: 12).

Information regarding all motor vehicle collisions that occurred between January 1994 and June 2000 wherein the study subject was the driver was obtained from the Alabama Department of Public Safety. Information of specific relevance to the study was abstracted from hard-copy accident reports, including the date of the accident and whether the study subject was deemed to be at-fault according to the officer at the scene.

The Institutional Review Board for Human Use at UAB approved the study protocol. The study followed the tenets of the Declaration of Helsinki and informed consent was obtained from the subjects after explanation of the nature and possible consequences of the study.

\section{Study Design}

Within the study cohort, a nested case-control study was conducted. Cases were those enrollees who experienced a MVC during the observation period. Incidence density sampling was used to select a single control for each case. Controls were subjects who, at the time of their selection, had not been involved in an MVC. In order for a control to be eligible for selection for a given case, the enrollee must have had their first visit during the chart abstraction period (i.e., between January 1994 and December 1995) prior to the date of the MVC. This ensured that both the case and their matched control had contemporaneous data. Among the eligible controls for a given case, a single patient was randomly selected. 
A total of 406 patients met the inclusion criteria for this study. During the observation period (i.e., January 1994-June 2000), a total of 120 collisions were observed and selected as cases of the study; of these collisions, $66(68.0 \%)$ were determined at the collision scene to be caused by the patient (at-fault cases). Regarding the amount of accidents each individual had, 75 had one accident, 18 had two accidents, and 4 had three accidents. For these 120 cases, a total of 120 controls were selected.

\section{Statistical Analysis}

Demographic, behavioral, driving and general health characteristics were not available for $40 \%$ of the selected cases and $37 \%$ of the selected controls due to failure to complete the telephone survey. Reasons for failing to complete the survey include death of the study subject, failure to contact the study subject following multiple attempts and refusal to participate. To prevent the exclusion of these subjects from the analysis, multiple imputation was used to create values for the missing observations using a Markov Chain Monte Carlo (MCMC) method (Rubin \& Schenker, 1991; Schafer, 1997; Allison, 2001) as described previously (McGwin, et al., 2004). This method was appropriate because the pattern of missing data tended to be monotone (i.e., for a given subject, groups of variables were missing information).

Descriptive statistics were generated for demographic, behavioral, driving and clinical characteristics. These variables were then compared between the case and control groups using $\chi^{2}$ and t-tests for categorical and continuous variables, respectively. Logistic regression was used to calculate crude and adjusted odds ratios (OR) and associated 95\% confidence intervals (CI) for the association between field defects and the risk of MVC involvement. Separate analyses were conducted using the AGIS score from the better and worse eye (defined according to the AGIS score). Adjusted analyses were used to account for the possible confounding effects of demographic and medical characteristics. Finally, analyses were also conducted limiting the case group to those patients who were deemed to be at-fault in the motor vehicle collision.

\section{RESULTS}

Table 1 presents the distribution of demographic, medical, and visual function characteristics among cases and controls; comparisons with at-fault cases are also presented.

Table 2 presents the crude and adjusted OR for MVC according to AGIS categories for the cases and controls. Overall, as the AGIS score increased (visual field defect was more severe) for both the better and worse eye, there was a corresponding increase in the odds of a MVC. Regarding the better eye, patients with a severe defect were more likely to have had a MVC than patients with no field defect, but this association was not statistically significant after adjustment for variables in Table 1. Regarding the worse eye, patients with a moderate or severe defect were more likely to subsequently have a MVC compared to patients with no field defect, for both the crude (OR 3.0 95\% CI 1.3-7.1 and OR 4.3 95\% CI 1.8-10.3, respectively) and adjusted analyses (OR 3.6 95\% CI 1.4-9.4 and 4.4 95\% CI 1.6-12.4, respectively).

Table 3 presents the crude and adjusted ORs for MVC according to AGIS score categories for the at-fault cases and controls. Similar to Table 2, as the AGIS score increased for the worse eye, there was a corresponding increase in the odds of a MVC; this was only the case for the better 
eye. Patients with a moderate or severe defect in the worse eye were more likely (OR $3.095 \% \mathrm{CI}$ 1.1-8.3 and 5.8 95\% CI 2.2-15.8, respectively) to have an at-fault crash than patients with no field defect. After adjustment, these associations were still significant for both moderate and severe defects (OR 4.0 95\% CI 1.1-13.8 and 6.7 95\% CI 1.9-23.7, respectively).

Table 1. Demographic, medical, and visual function characteristics among glaucoma patients in a MVC (cases) versus those not (controls) and those at fault for a MVC versus controls

\begin{tabular}{|c|c|c|c|c|c|c|}
\hline & $\begin{array}{c}\text { Cases } \\
(n=120)\end{array}$ & $\begin{array}{c}\text { Controls } \\
(n=120)\end{array}$ & p-value & $\begin{array}{c}\text { At-fault } \\
\text { Cases } \\
(n=84) \\
\end{array}$ & $\begin{array}{c}\text { Controls } \\
(n=120)\end{array}$ & p-value \\
\hline Age, years, mean & 73.4 & 72.3 & 0.2348 & 72.7 & 72.3 & 0.0635 \\
\hline Gender, \% & & & 0.0037 & & & 0.0001 \\
\hline Male & 56.9 & 38.3 & & 65.5 & 38.3 & \\
\hline Female & 43.1 & 61.7 & & 34.5 & 61.7 & \\
\hline Race, $\%$ & & & 0.1160 & & & 0.7723 \\
\hline Caucasian & 64.1 & 73.7 & & 71.8 & 73.7 & \\
\hline African-American & 35.9 & 26.3 & & 28.2 & 26.3 & \\
\hline Ever smoke, $\%$ & 34.2 & 25.0 & 0.1185 & 27.4 & 25.0 & 0.7027 \\
\hline Ever consume alcohol, \% & 47.5 & 40.0 & 0.2609 & 55.9 & 40.0 & 0.0246 \\
\hline \multicolumn{7}{|l|}{ Medical conditions, $\%$} \\
\hline Cataract & 88.6 & 77.5 & 0.0207 & 92.9 & 77.5 & 0.0034 \\
\hline Diabetic retinopathy & 32.5 & 23.3 & 0.1107 & 47.6 & 23.3 & 0.0003 \\
\hline Age-related maculopathy & 29.3 & 30.8 & 0.7902 & 41.7 & 30.8 & 0.1110 \\
\hline Hearing aid use & 33.3 & 33.3 & 0.9999 & 46.4 & 33.3 & 0.0588 \\
\hline Fall & 49.6 & 48.3 & 0.8442 & 63.1 & 48.3 & 0.0372 \\
\hline No. glaucoma medications, mean & 4.03 & 3.89 & 0.5219 & 3.94 & 3.89 & 0.8338 \\
\hline Cognitive impairment, $\%$ & 3.13 & 3.35 & 0.6239 & 3.87 & 3.35 & 0.2933 \\
\hline \multicolumn{7}{|l|}{ Acuity, $\log M A R$, mean } \\
\hline Better eye & 0.24 & 0.22 & 0.4761 & 0.20 & 0.22 & 0.2518 \\
\hline Worse eye & 0.25 & 0.21 & 0.1269 & 0.20 & 0.21 & 0.1967 \\
\hline Driving avoidance score, mean & 2.20 & 2.87 & 0.0270 & 2.58 & 2.87 & 0.3942 \\
\hline Miles driven per year, mean & 7479 & 9784 & 0.0298 & 8395 & 9784 & 0.2420 \\
\hline
\end{tabular}


Table 2. Crude and adjusted OR according to AGIS score categories for cases and controls

\begin{tabular}{|c|c|c|c|c|c|c|}
\hline & $\begin{array}{c}\text { Cases } \\
(\mathrm{n}=120)\end{array}$ & $\begin{array}{l}\text { Controls } \\
(\mathrm{n}=120)\end{array}$ & $\begin{array}{c}\text { Crude } \\
\text { OR }(95 \% \text { CI) } \\
\end{array}$ & p-value & $\begin{array}{c}\text { Adjusted* } \\
\text { OR (95\% CI) }\end{array}$ & p-value \\
\hline Better eye & $\%$ & $\%$ & & & & \\
\hline No Defect & 33.3 & 44.2 & Reference & & Reference & \\
\hline Mild defect & 38.2 & 35.8 & $1.4(0.8,2.5)$ & 0.2434 & $1.5(0.7,2.8)$ & 0.2632 \\
\hline Moderate defect & 17.9 & 15.0 & $1.6(0.7,3.3)$ & 0.2285 & $1.4(0.5,3.4)$ & 0.5089 \\
\hline Severe defect & 10.6 & 5.0 & $2.8(1.0,8.0)$ & 0.0545 & $3.2(0.9,10.4)$ & 0.0576 \\
\hline \multicolumn{7}{|l|}{ Worse eye } \\
\hline No defect & 10.0 & 21.1 & Reference & & Reference & \\
\hline Mild defect & 25.8 & 37.4 & $1.5(0.6,3.3)$ & 0.3669 & $1.3(0.5,3.4)$ & 0.5361 \\
\hline Moderate defect & 31.7 & 22.0 & $3.0(1.3,7.1)$ & 0.0096 & $3.6(1.4,9.4)$ & 0.0099 \\
\hline Severe defect & 35.0 & 17.1 & $4.3(1.8,10.3)$ & 0.0009 & $4.4(1.6,12.4)$ & 0.0045 \\
\hline
\end{tabular}

* Adjusted for all variables in Table 1.

Table 3. Crude and adjusted OR according to AGIS score categories for at-fault cases and controls

\begin{tabular}{lcccccc}
\hline & $\begin{array}{c}\text { Cases } \\
(\mathbf{n = 8 4})\end{array}$ & $\begin{array}{c}\text { Controls } \\
(\mathbf{n = 1 2 0})\end{array}$ & $\begin{array}{c}\text { Crude } \\
\text { OR } \mathbf{( 9 5 \%} \mathbf{C I})\end{array}$ & $\begin{array}{c}\text { Adjusted }^{*} \\
\text { p-value } \\
\text { OR (95\% CI) }\end{array}$ & p-value \\
\hline Better eye & & & & & & \\
& & & & & & \\
No Defect & 33.3 & 44.2 & Reference & & Reference & \\
Mild defect & 36.9 & 35.8 & $1.4(0.7,2.6)$ & 0.3488 & $1.4(0.6,3.1)$ & 0.4176 \\
Moderate defect & 20.2 & 15.0 & $1.8(0.8,4.0)$ & 0.1576 & $1.6(0.6,4.5)$ & 0.3903 \\
Severe defect & 9.5 & 5.0 & $2.5(0.8,8.0)$ & 0.1157 & $2.8(0.7,11.1)$ & 0.1397 \\
& & & & & & \\
Worse eye & & & & & & \\
& & & & & & \\
No defect & 8.3 & 21.1 & Reference & & \\
Mild defect & 26.2 & 37.4 & $1.8(0.7,4.7)$ & 0.2491 & $1.4(0.5,4.6)$ & 0.5278 \\
Moderate defect & 26.2 & 22.0 & $3.0(1.1,8.3)$ & 0.0311 & $4.0(1.1,13.8)$ & 0.0306 \\
Severe defect & 39.3 & 17.1 & $5.8(2.2,15.8)$ & 0.0005 & $6.7(1.9,23.7)$ & 0.0033 \\
& & & & & & \\
\hline
\end{tabular}

* Adjusted for all variables in Table 1.

\section{DISCUSSION}

Older adults with glaucoma with moderate to severe central field loss in their worse functioning eye are at increased risk for collision involvement as compared to those with glaucoma who have no field loss. In this case-control study, those with AGIS scores indicating severe central field impairment were 6 times more likely to incur an at-fault crash and 4 times more likely to incur a crash regardless of fault than were those with AGIS scores indicating no impairment. Previous studies on drivers with visual field loss are consistent with these findings, although they did not focus on glaucomatous drivers per se and used measurement methods quite different than ours. Drivers with severe binocular field loss as determined by a screening test administered $40^{\circ}$ 
nasally and $60^{\circ}$ temporally at motor vehicle licensing offices were about two times more likely to be crash involved compared to those with no field loss (Johnson \& Keltner, 1983). In our study, the visual field variable was not based on a screening test but rather on a full-threshold procedure in the central field. A study on driving performance on a closed road-course found that the avoidance of obstacles was impaired for normally sighted persons with simulated field loss reducing the diameter of the field to a $30^{\circ}$ radius (Wood \& Troutbeck, 1992).

In the present study, visual field loss was defined in terms of each eye separately, as is the clinical convention when managing glaucoma and monitoring its potential progression. One might argue based on face validity that it is the binocular visual field assessed by conventional automated perimetry methods or by the Esterman grid (Esterman, 1983), not the monocular central field of each eye, that is the most direct way to evaluate risk for the adverse events such as falling, problems locating objects, and vehicle crashes; however this conjecture remains to be proven. In clinical practice, each eye's health and functionality is routinely evaluated separately. In view of this, it is important to recognize, based on this study's results, that the convention of assessing each eye's field by itself, even just the central field, is informative with respect to crash risk. It is further interesting that the worse eye's field sensitivity was more strongly related to crash risk than that for the better eye. There is a conventional notion in clinical practice that visual performance is dictated by the eye with better function. However, the worse eye's visual field's characteristics were significantly associated with crash involvement, whereas those of the better eye were not. These findings are reminiscent of our earlier findings on contrast sensitivity and crash risk in drivers with cataract, where severe contrast sensitivity impairment in one eye only elevated crash risk (Owsley, et al., 2001).

Strengths of this study are as follows. The medical records of all study subjects were reviewed to confirm glaucoma as the primary eye disorder and cause of visual dysfunction. This is important because other eye diseases common in the elderly (e.g., diabetic retinopathy, age-related macular degeneration) can cause central visual field impairment. Second, the AGIS score was used to define severity of field defect. The AGIS score is an already established metric of field loss severity in glaucoma and is computed based on the output of the most common automated perimetry programs $(30-2,24-2)$ used in glaucoma management. Third, an independent and impartial source (Alabama Department of Public Safety) was used to obtain information on MVCs for the study participants; crash data were not based on driver self-reports known to be unreliable (McGwin, Owsley \& Ball, 1998). Procurement of the accident report also allowed us to obtain information on who was responsible for the MVC and therefore refine our case definition to focus on those MVCs in which the study subject was deemed at-fault.

Study limitations must also be acknowledged. General health and driving habits were collected in 2000 using a telephone survey that relied on participants' ability to recall these characteristics with 1995 as the reference point. However, there is little reason to suspect differential bias among the cases and controls in the ability to accurately recall the requested information, and thus any misclassification is likely to result in a conservative bias. Second, the response rate for the telephone survey was not ideal (approximately 61\% overall due), yet it did not differ between the cases $(60 \%)$ and controls $(63 \%)$. For those who did not complete the survey, multiple imputation was used to create values for the missing information. Fortunately, the primary independent variable (i.e., visual field defect) was obtained from each patient's medical record and therefore not vulnerable to survey non-response. Moreover, when the adjusted analyses were 
run excluding patients with imputed data, the overall results were highly consistent with the results based upon all patients, suggesting little bias resulting from the imputation process.

In conclusion, this study suggests that glaucomatous drivers age 55 and over with moderate to severe impairment in the central $24^{\circ}$ field of their worse functioning eye are at increased risk for vehicle crash involvement. This finding has clinical relevance for ophthalmologists caring for patients with glaucoma, since it provides some guidance as to what point in disease progression it is prudent to begin a dialogue about driver safety between doctor and patient. What is practically useful is that the marker for increased crash risk identified here can be computed from the automated perimetry tests commonly used in the management of glaucoma, and thus there is no additional patient or economic burden.

\section{ACKNOWLEDGMENTS}

This research was supported by National Institutes of Health grants P50-AG11684 and R21EY14071, Research to Prevent Blindness, Inc., and the EyeSight Foundation of Alabama. Cynthia Owsley is a Research to Prevent Blindness Senior Scientific Investigator.

\section{REFERENCES}

Advanced Glaucoma Intervention Study. (1994). Visual field test scoring and reliability. Opthamology 101:1445-1455.

Allison, PD. (2001). Missing Data. Thousand Oaks, CA: Sage.

Esterman, B. (1983). Functional scoring of the binocular visual field. In: Greve, E.L. \& Heijl, A. (Eds.) Fifth International Visual Field Symposium. The Hague, The Netherlands: Dr W. Junk Publishers: 187-192.

Gutierrez, P., Wilson, M.R., Johnson, C., Gordon, M., Cioffi, G.A., Ritch, R., et al. (1997). Influence of glaucomatous visual field loss on health-related quality of life. Arch Ophthalmol 115: $777-784$.

Hu, P.S., Trumble, D.A., Foley, D.J., Eberhard, J.W., Wallace, R.B. (1998). Crash risks of older drivers: a panel data analysis. Accid Anal Prev. 30(5):569-581.

Johnson, C.A., Keltner, J.L. (1983). Incidence of visual field loss in 20,000 eyes and its relationship to driving performance. Arch Ophthalmol. 101:371-375.

McCloskey, L.W., Koepsell, T.D., Wolf, M.E., Buchner, D.M. (1994). Motor vehicle collision injuries and sensory impairments of older drivers. Age Ageing 23(4): 267-273.

McGwin, G. Jr., Mays, A., Joiner, W., Decarlo, D.K., McNeal, S., Owsley, C. (2004). Is glaucoma associated with motor vehicle collision involvement and driving avoidance? Invest Ophthalmol Vis Sci. 45: 3934-3939.

McGwin, G. Jr., Owsley, C., Ball, K. (1998). Identifying crash involvement among older drivers: Agreement between self-report and state records. Accid Anal Prev. 30: 781-791.

Murakami, E., Wagner, D.P. (1997). Comparison between computer-assisted self-interviewing using GPS with retrospective trip reporting using telephone interviews. Washington, DC: Federal Highway Administration, US Department of Transportation. 
Owsley, C., McGwin, G. Jr., Ball, K. (1998). Vision impairment, eye disease, and injurious motor vehicle crashes in the elderly. Ophthalmic Epidemiol. 5: 101-113.

Quigley, H.A. (1996). Number of people with glaucoma worldwide. Br J Ophthalmol. 80: 389393.

Wood, J.M., Troutbeck, R. (1992). Effect of restriction of the binocular visual field on driving performance. Ophthalmic Physiol Opt. 12: 291-298.

Owsley, C., McGwin, G. Jr., Sloane, M.E., Wells, J., Stalvey, B.T., Gauthreaux, S. (2002). Impact of cataract surgery on motor vehicle crash involvement by older adults. JAMA. 288: 841-849.

Owsley, C., Stalvey, B.T., Wells, J., Sloane, M., McGwin, G. Jr. (2001). Visual risk factors for crash involvement in older drivers with cataract. Arch Ophthalmol. 119: 881-887.

Owsley, C., Stalvey, B., Wells, J., Sloane, M.E. (1999). Older drivers and cataract: driving habits and crash risk. J Gerontol A Biol Sci Med Sci. 54A(4): M203-M211.

Pfeiffer, E. (1975). A short portable mental status questionnaire for the assessment of organic brain deficit in elderly patients. J Am Geriatr Soc. 23(10): 433-41.

Roccaforte, W.H., Burke, W.J., Bayer, B.L., Wengel, S.P. (1994). Reliability and validity of the Short Portable Mental Status Questionnaire administered by telephone. J Geriatr Psychiatry Neurol, .7: 33-38.

Rubin, D.B, Schenker, N. (1991). Multiple imputation in health-care databases: an overview and some applications. Stat Med. 10: 585-598.

Schafer, J.L. (1997). Analysis of Incomplete Multivariate Data. London: Chapman and Hall. 